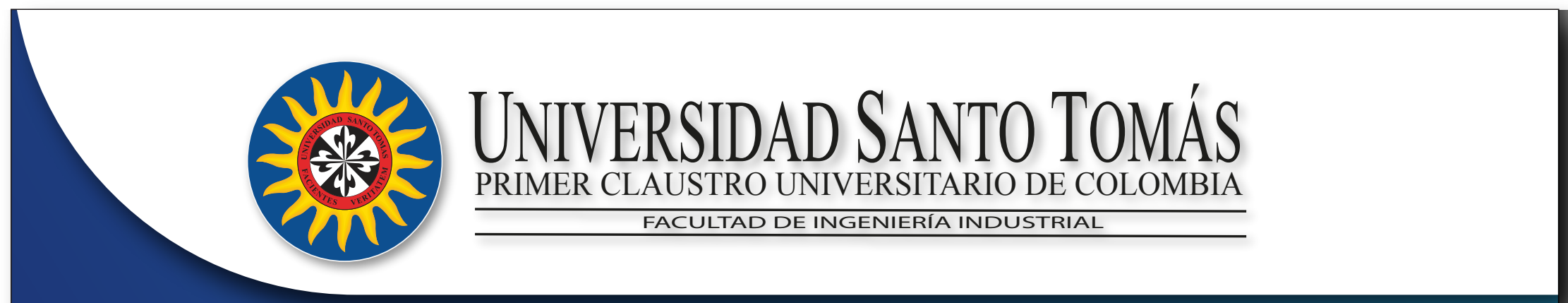

\title{
Cartilla de ensamble Campero Jeep
}

Elaborado por:

Natalia Díaz L.

Alejandro Pérez S.

Líder Investigador:

Óscar Emmanuel Granados Delgado
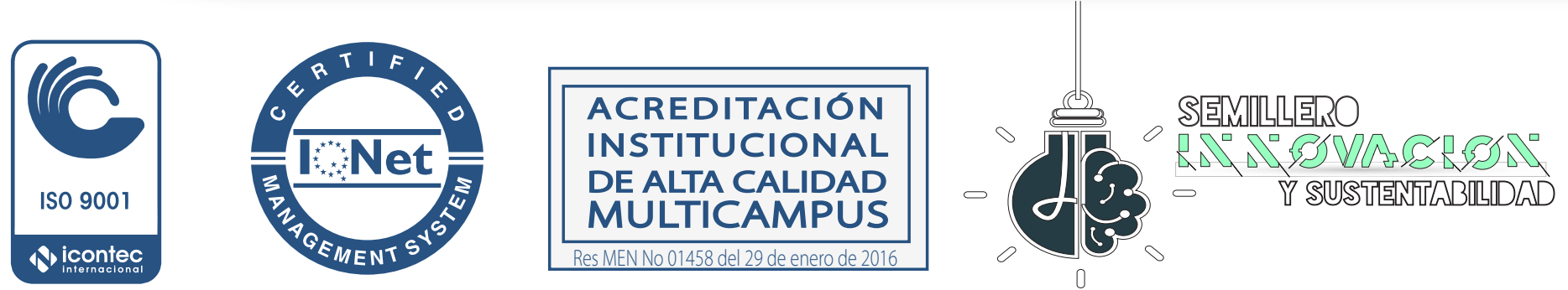


\section{CONTENIDO}

1. INTRODUCCIÓN

2. OBJETIVOS

a. Objetivo General

b. Objetivos Específicos

3. METOdología

4. DIAGRAMA DE FLUJO

5. PARTES DE ENSAMBLE

6. PROCEDIMIENTO

7. RESULTADOS

8. CONCLUSIONES
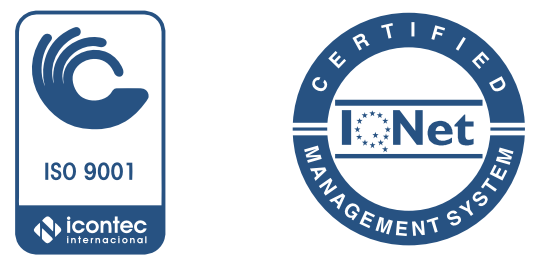


\section{INTRODUCCIÓN}

En un mundo global, donde el conocimiento varía cada segundo debido a la velocidad a la que avanza la tecnología, se requiere por parte de nuevos ingenieros habilidades que van más allá del conocimiento técnico, las ciencias básicas y otras materias teóricas.

La presente cartilla se realiza como resultado de un proceso experimental de diseño y manufactura de un producto de fabricación a escala, en este caso un carro - Jeep Wrangler, Sport 2018. La cartilla muestra el procedimiento de ensamble del carro a escala, producto del Proyecto Cooperativo de Ingeniería Industrial entre la Universidad Santo Tomás de Bogotá (Colombia) y Instituto Tecnológico de Costa Rica, con la finalidad de promover la generación de nuevos proyectos de trabajo en equipo a nivel continental, sin importar la distancia de los grupos y con el apoyo de las nuevas tecnologías de la comunicación.

La Universidad Santo Tomás, Sede Principal, fue representada en este proyecto por el Semillero de Investigación en Innovación y Sustentabilidad (SII2).
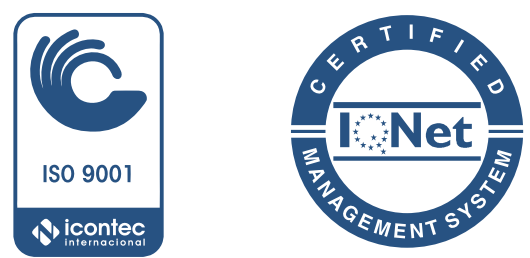

DEALTACALDAD 


\section{OBJETIVOS}

\section{Objetivo general}

Presentar el procedimiento de ensamble de un prototipo a escala del Jeep Wrangler Sport 2018 que permita estandarizar los procesos de diseño y fabricación entre la Universidad Santo Tomás y Tecnológico de Costa Rica.

\section{Objetivos especifficos}

- Identificar las diferentes piezas que hacen parte del proceso de ensamble del carro a escala.

- Relacionar el paso a paso del ensamble del carro a escala.
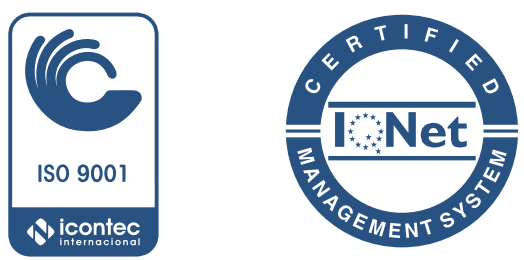


\section{METODOLOGÍA}

Una vez definido el modelo del carro a diseñar se realizó el modelamiento 3D en el software Innventor, seguido por el corte láser de las piezas en madera e impresión 3D de las piezas en PLA. Para establecer los lineamientos de ensamble del carro, se realizó una sesión por Skype la cual fue la herramienta seleccionada como canal de comunicación entre las dos universidades, lo que permitió definir los pasos estandarizados para el ensamble del carro modelo Jeep Wrangler 2018, como guía para cada uno de los grupos conformados, tanto de la Universidad Santo Tomás Sede Principal como del Tecnológico de Costa Rica.

\section{Palabras clave}

Fabricar, ensamblar, campero Jeep, carro a escala, estandarización.
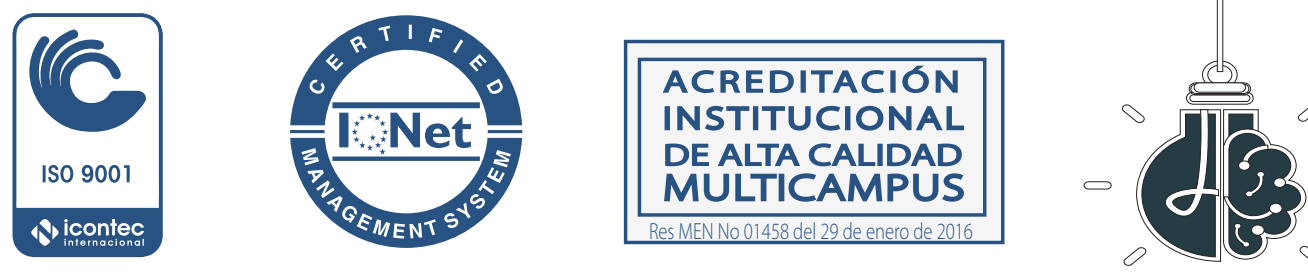

SEVIILIERO

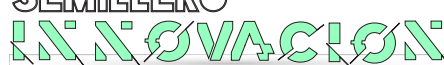




\section{DIAGRAMA DE FLUJO}
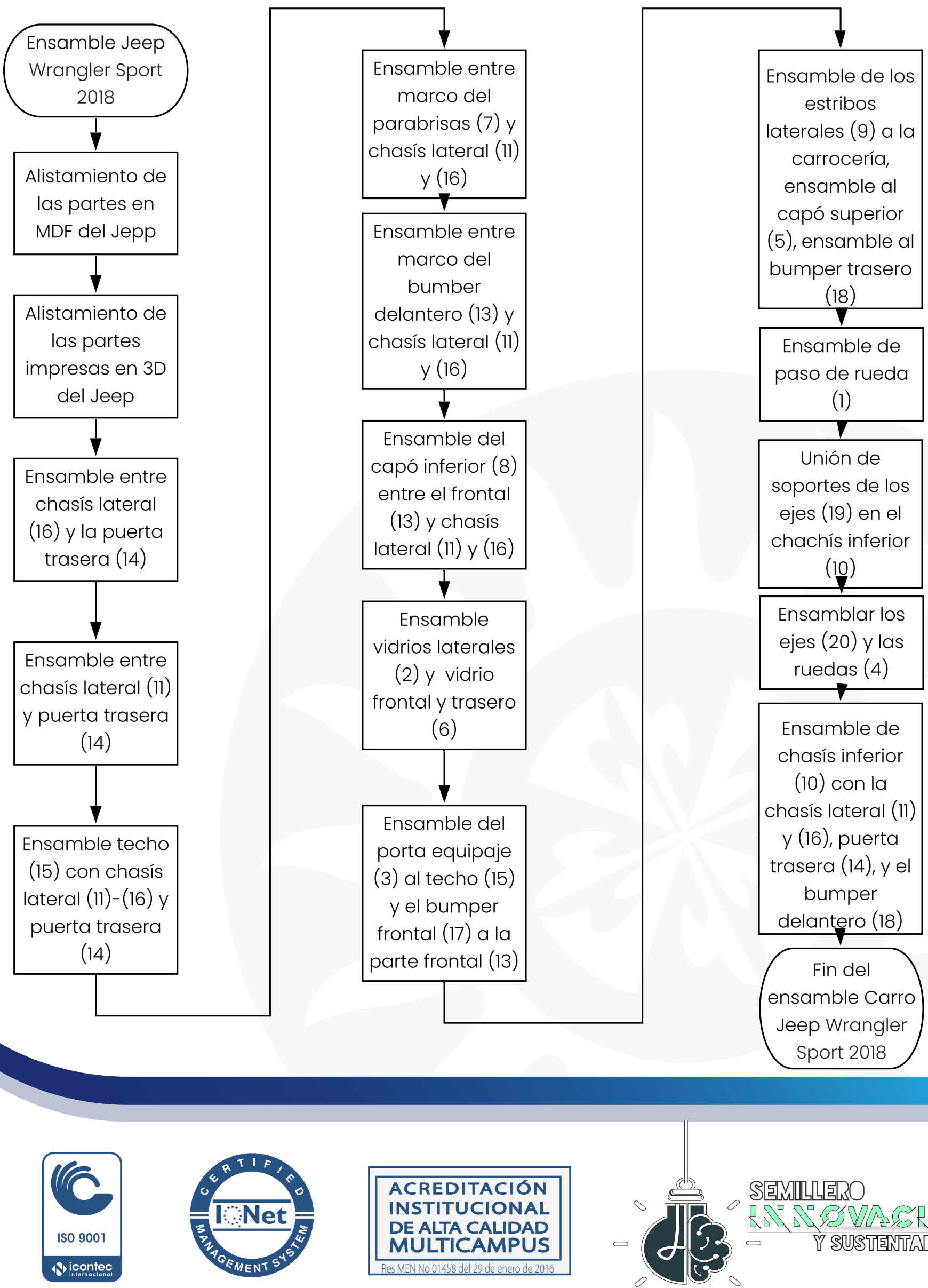

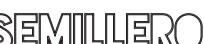

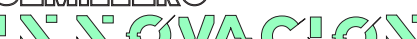




\section{PARTES DE ENSAMBLE}

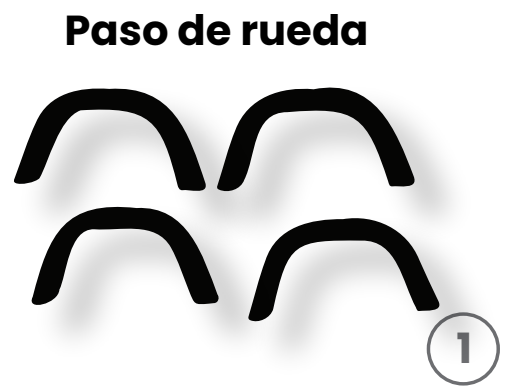

Capó superior

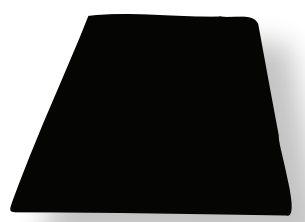

Estribos laterales

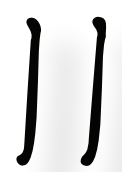

Frontal

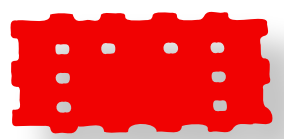

(13)
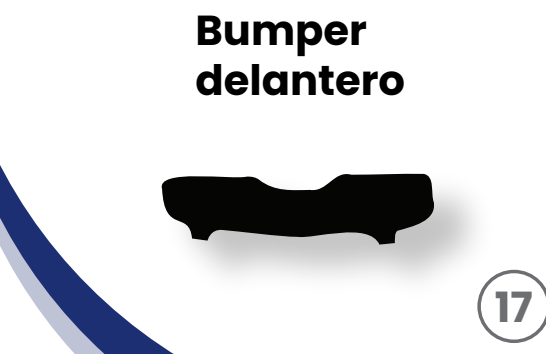

(17)

(9)

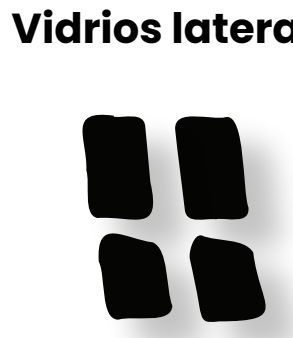

(2)
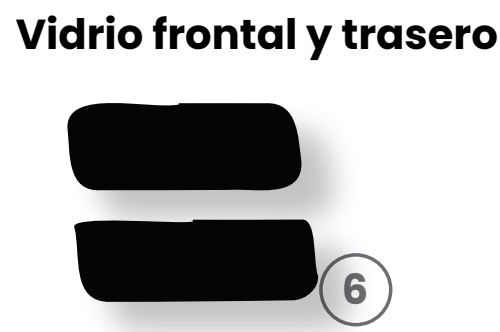

Chasís inferior
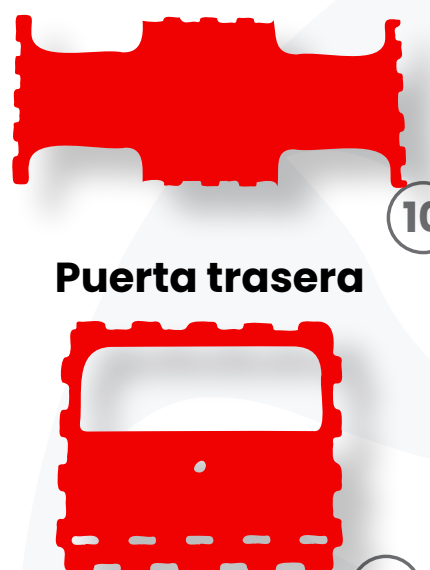

(14)
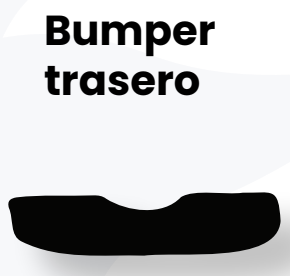

(18)
Porta equipaje

Ruedas

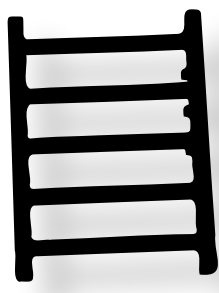

(3)

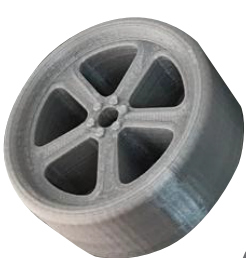

(4)
Marco vidrio frontal (panorámico)

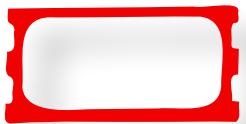

(7)

Chasís lateral

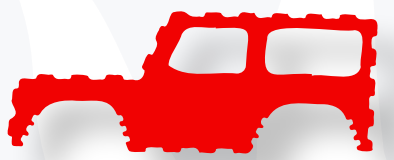

(11)

Techo

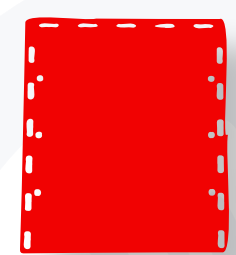

(15)
Capó Inferior

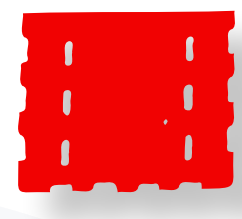

Espejos

a

(12)

Chasís lateral

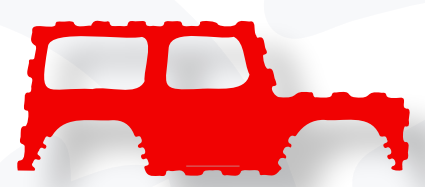

(16)

Ejes
Soportes ejes

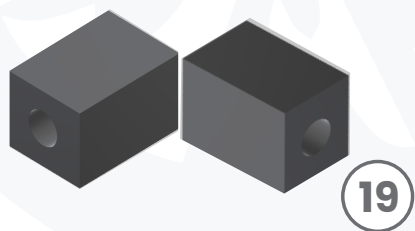

(19)
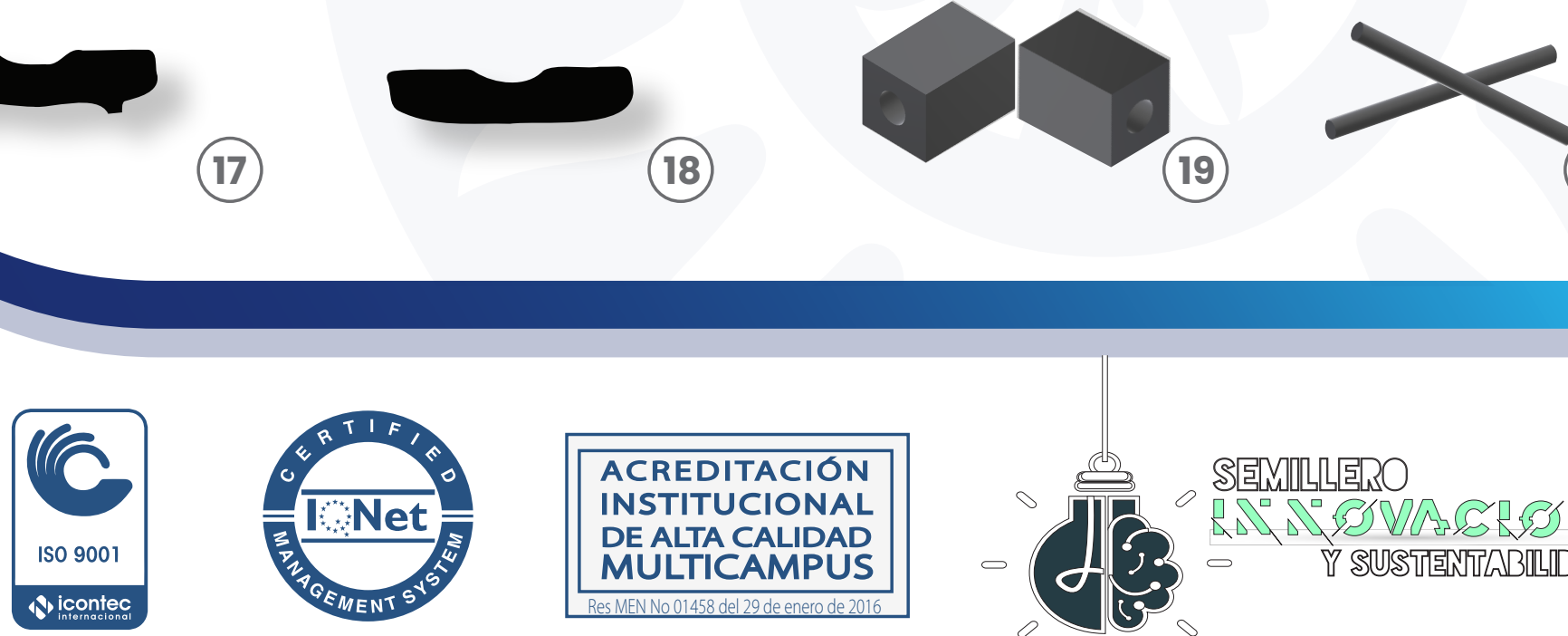

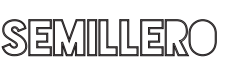

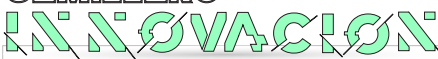




\section{PROCEDIMIENTO}

Para el ensamble del campero se recomienda usar pegante para madera (colbón) en las partes del MDF (partes rojas) y para las partes en plástico PLA (partes negras), recomendamos usar algún tipo de silicon ligero no muy espeso.

1) Se realiza el ensamble entre chasís lateral (16) y la puerta trasera (14)

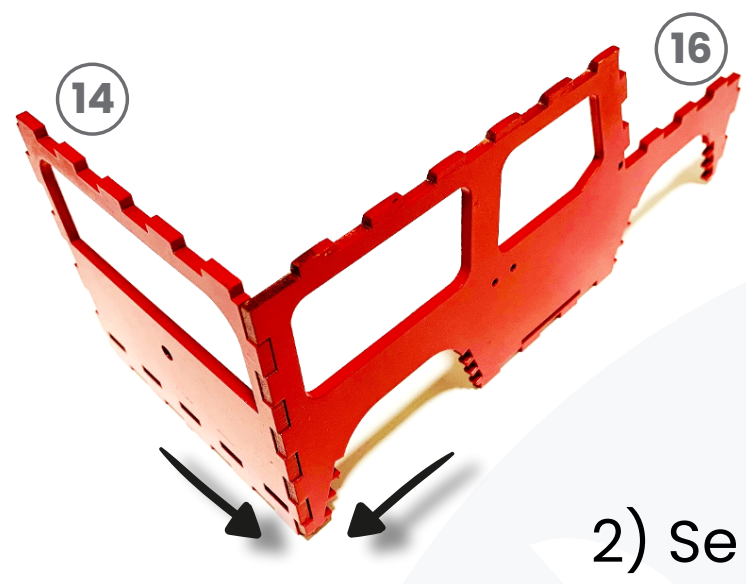

lateral (11) y la puerta trasera (14)
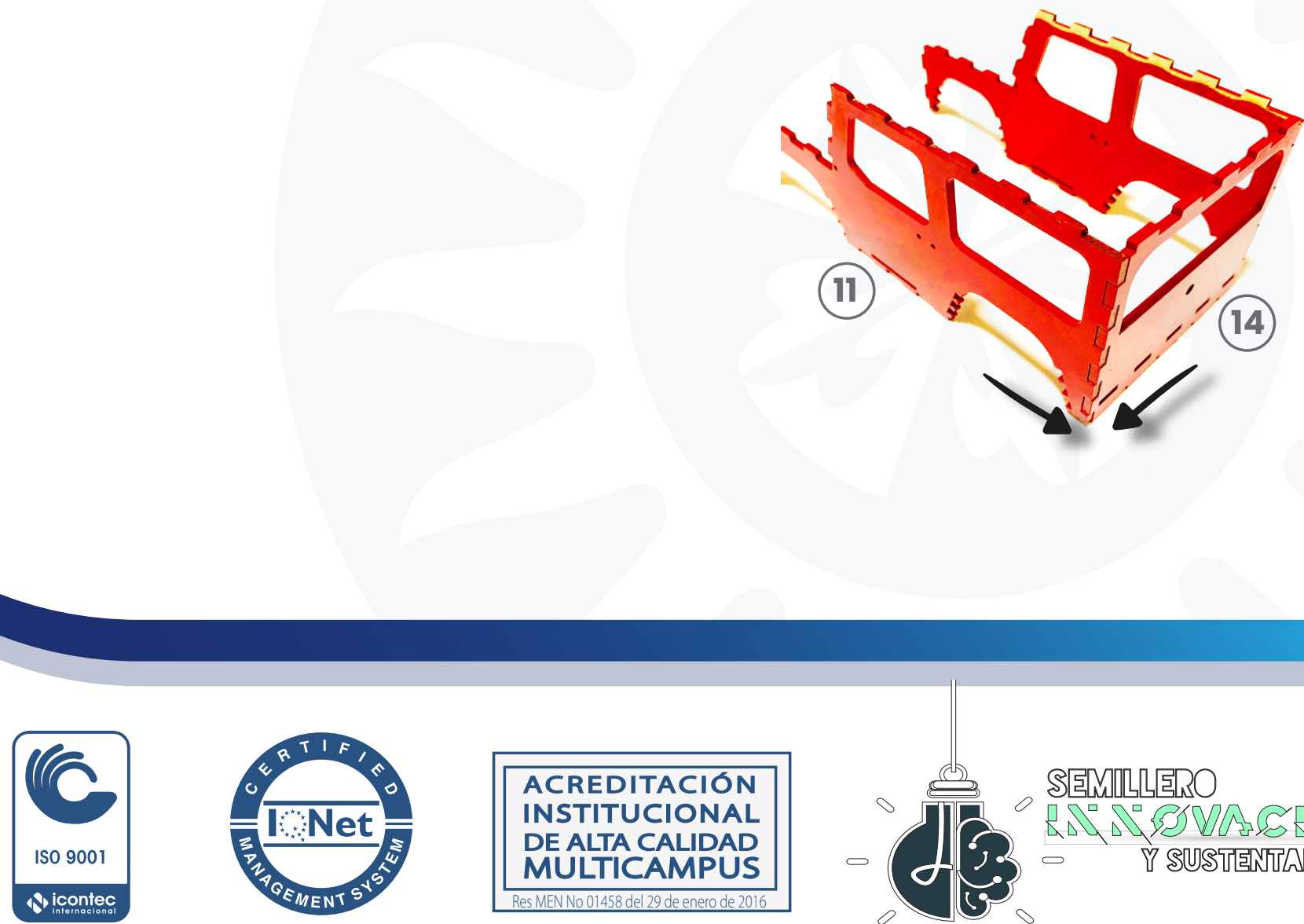


\section{Procedimiento}

3) Se realiza el ensamble del techo (15) con chasís lateral (11),(16) y puerta trasera (14).

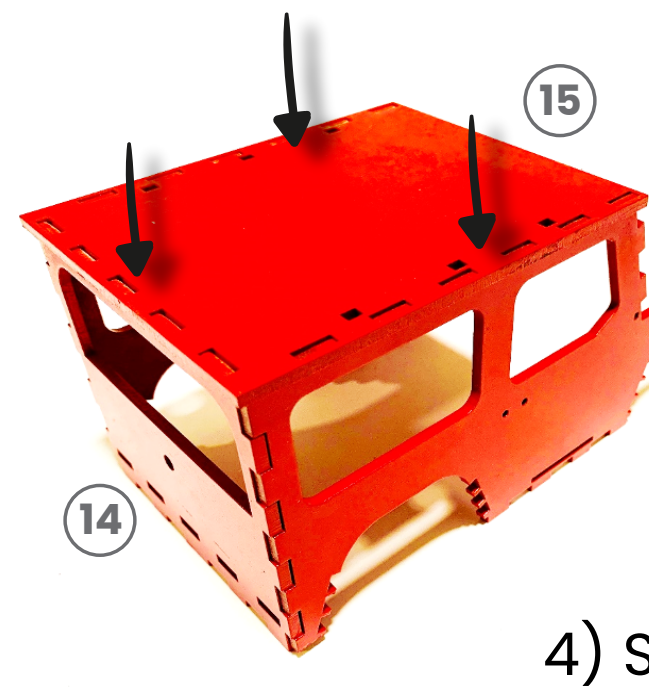

(16)

4) Se realiza el ensamble entre el marco del parabrisas (7) y chasís lateral (11) y (16).
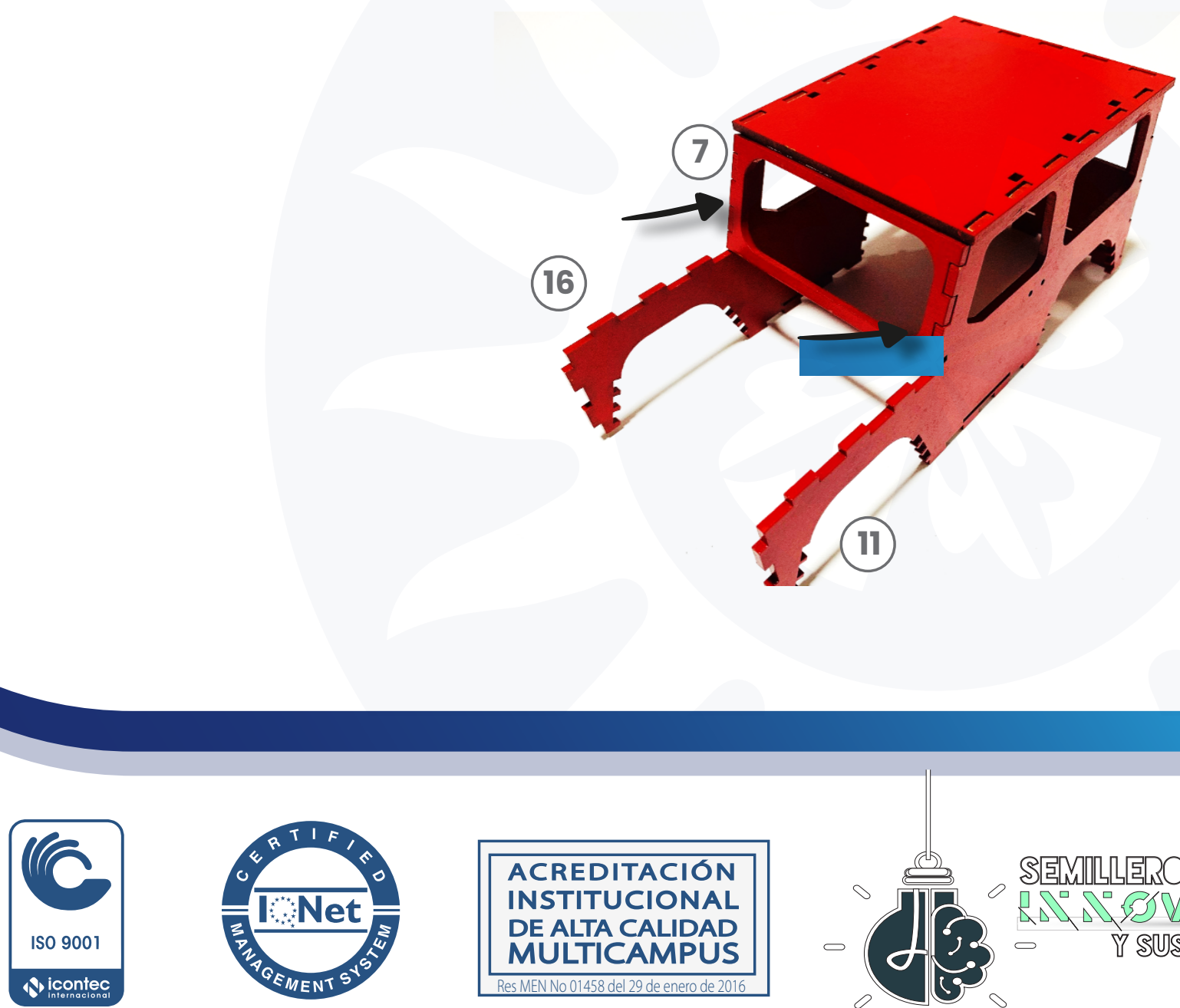


\section{Procedimiento}

5)Se realiza el ensamble entre el marco del bumper delantero (13) y chasís lateral (11) y (16).

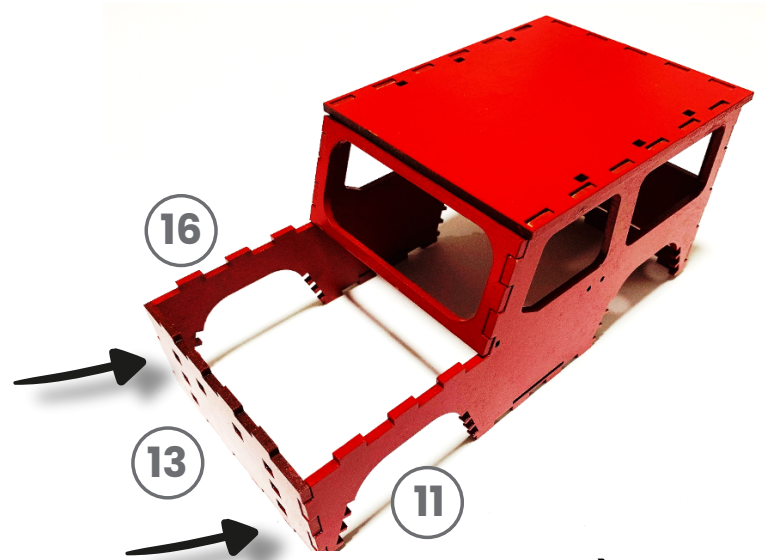

6) Se realiza el ensamble del capó inferior (8) entre el frontal (13) y chasís lateral (16) y (11).
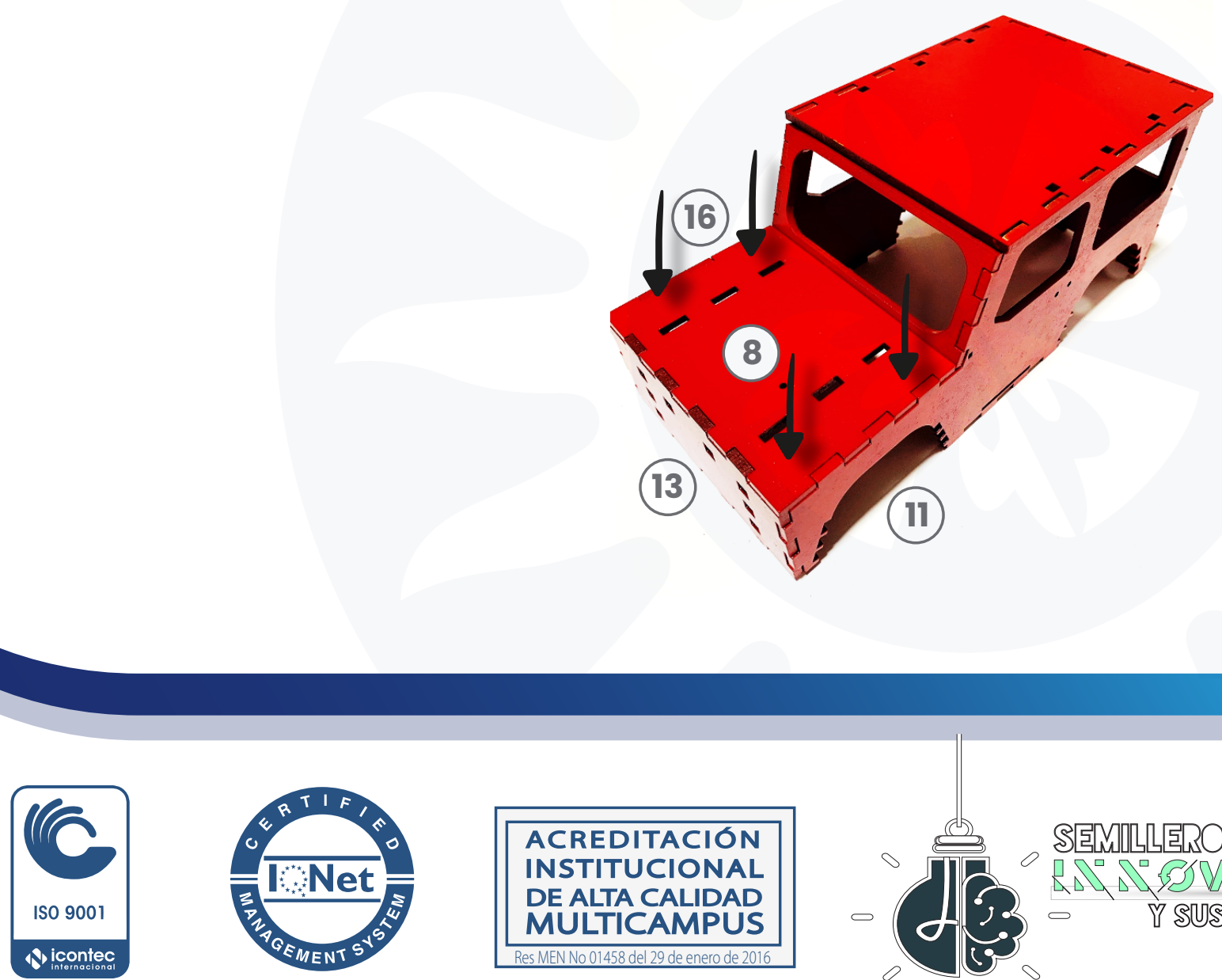


\section{Procedimiento}

7) Se realiza el ensamble de vidrios laterles

(2) y vidrio frontal y trasero (6).
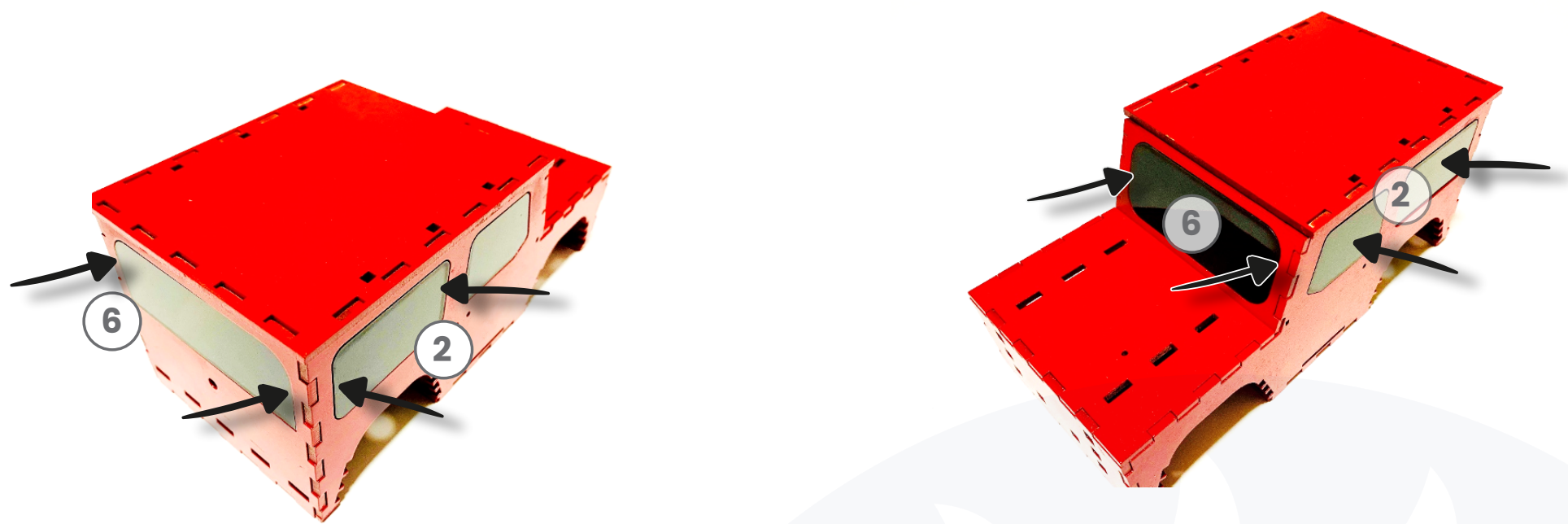

8) Se realiza el ensamble del porta equipaje (3) al techo (15) y el bumper delantero (17) a la parte frontal (13).
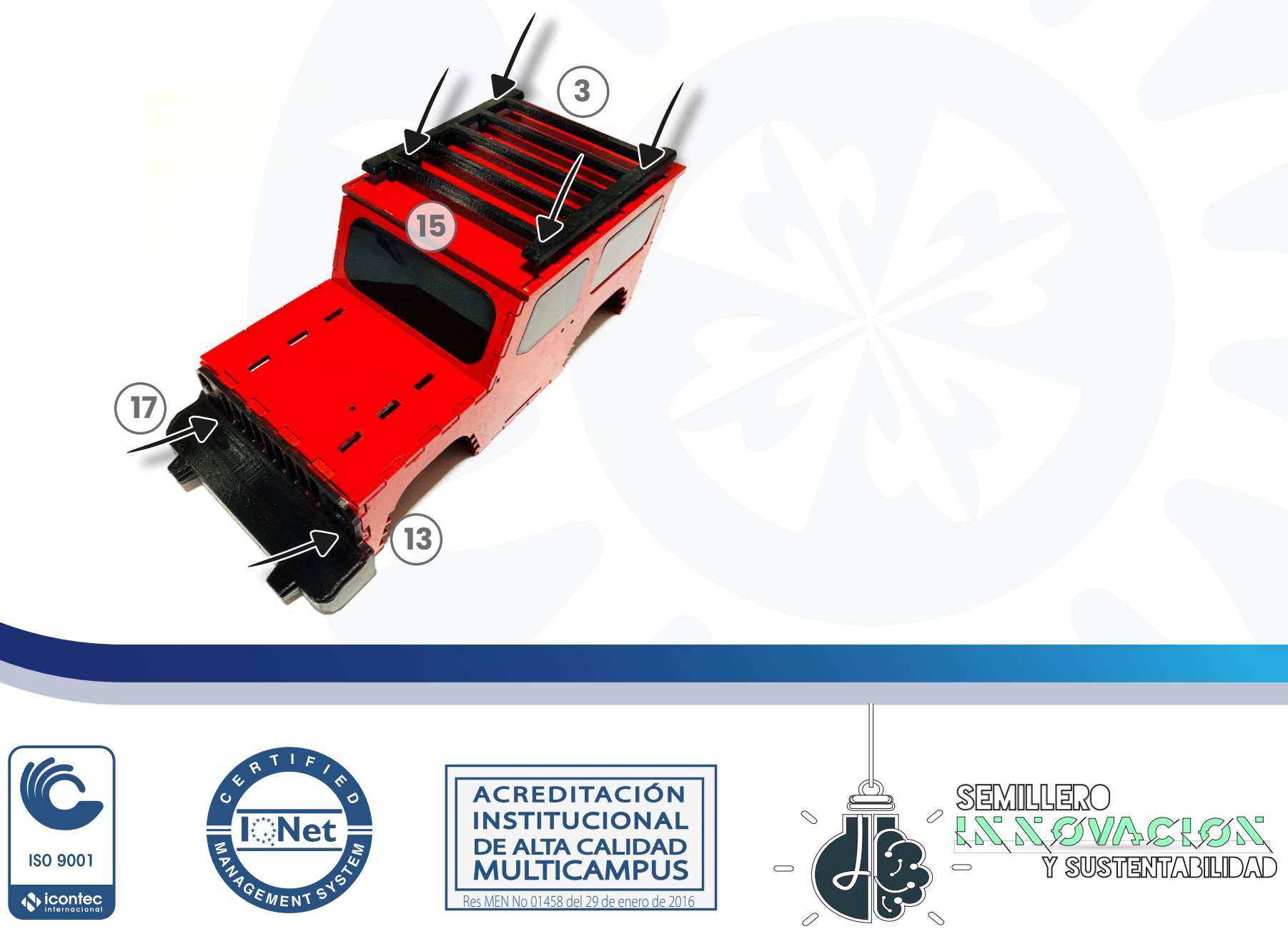


\section{Procedimiento}

9) Se realiza el ensamble de los estribos laterales (9) a la carroceria, ensamble de la tapa capó (5) y se realiza el ensamble del bumper trasero (18).
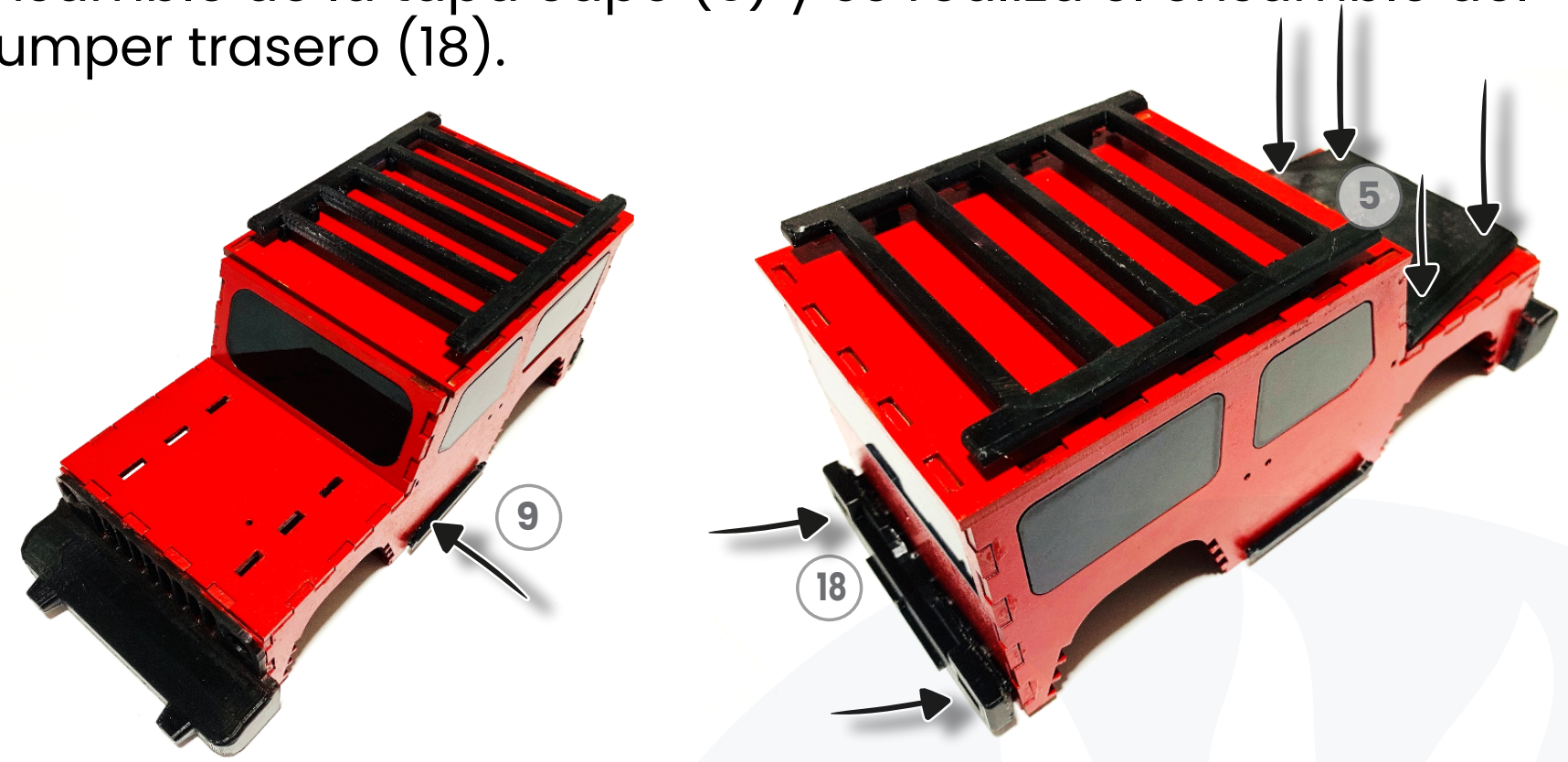

10) Se realiza el ensamble de paso de rueda (1).
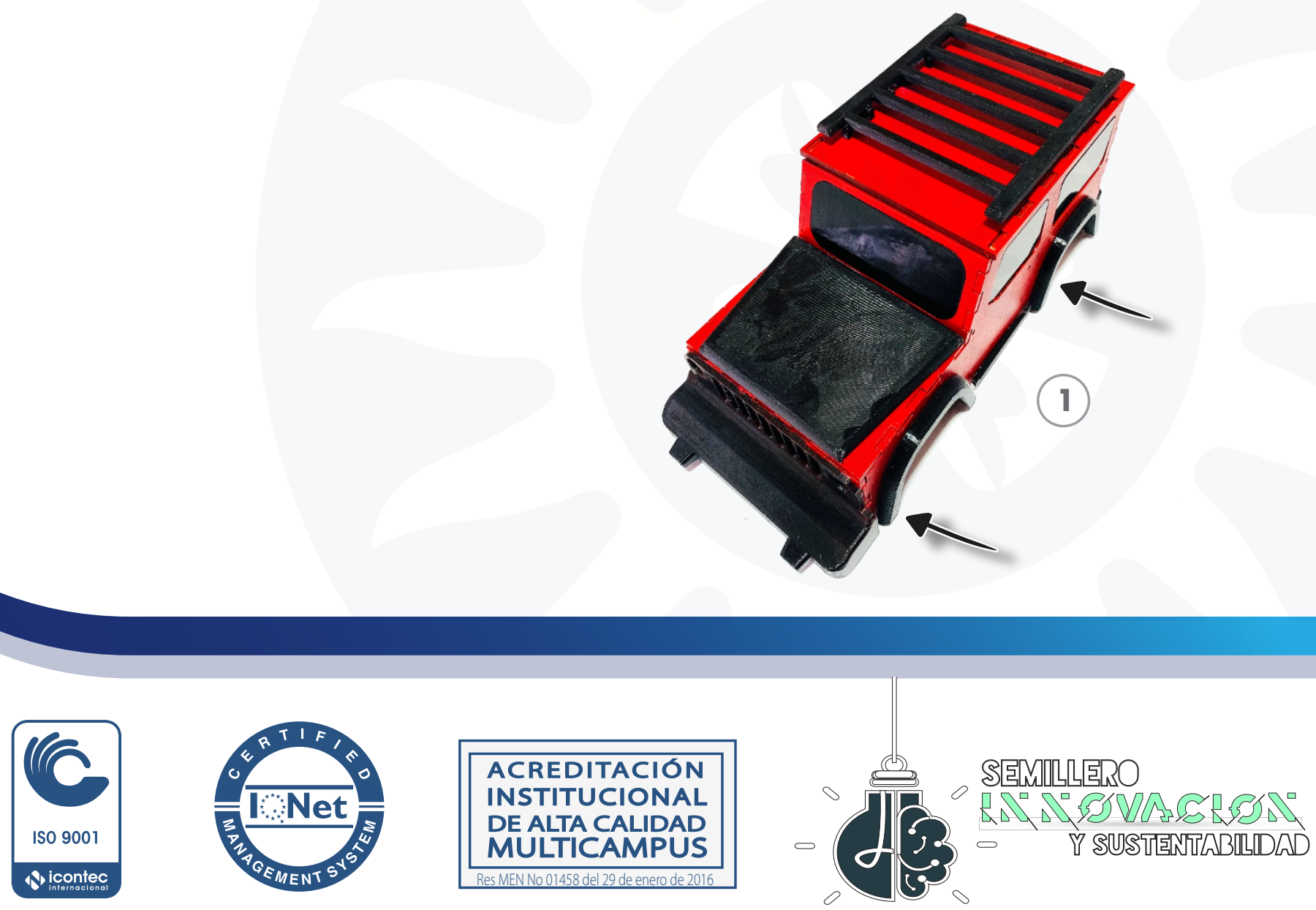


\section{Procedimiento}

11) Se realiza la unión de los soportes de los ejes (19) en el chasís inferior (10), luego ensamblar los ejes (20) y las ruedas (4).
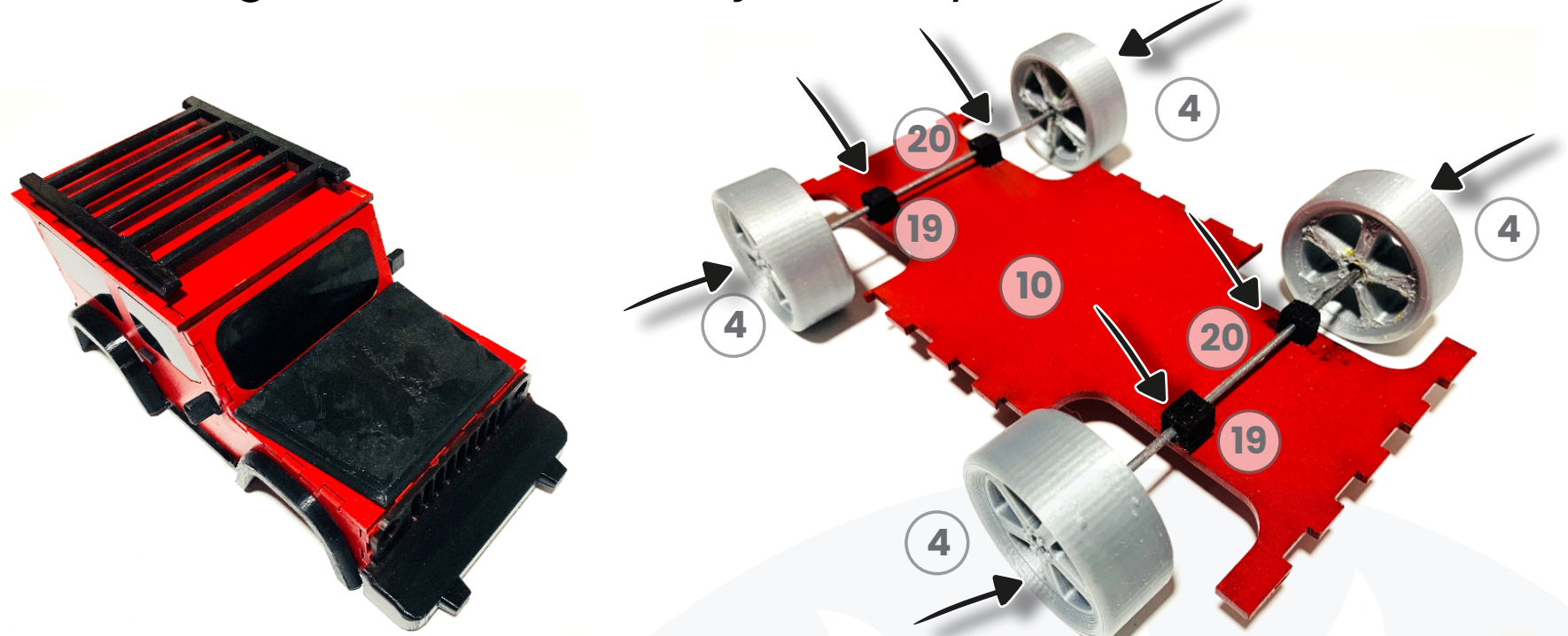

12) Se realiza el ensamble del chasís inferior (10) con la chasís lateral (11) y (16), puerta trasera (14), y el bumper delantero (18).
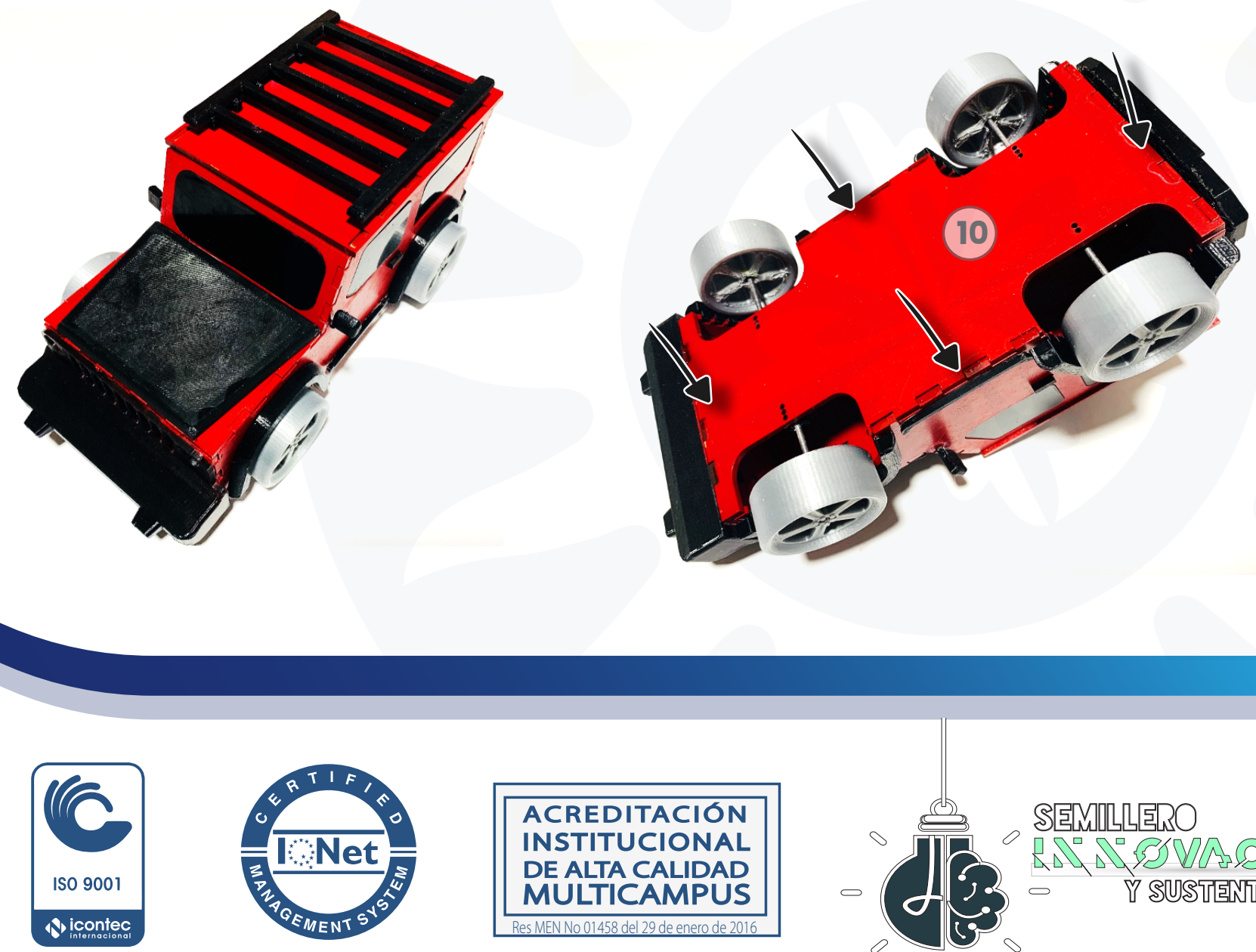


\section{RESULTADOS}

Se obtuvo la interacción entre los dos grupos que conformaban el proyecto, tanto de la Universidad Santo Tomás Sede Principal como del Tecnológico de Costa Rica, por medio de la herramienta de comunicación seleccionada Skype, realizando diferentes sesiones que permitieran despejar dudas y comentar sobre los problemas que fueron ocurriendo durante el proceso. Para el ensamble del carro se contó con una sesión que permitió evidenciar la construcción a escala del Jeep Wrangler 2018 por parte de los dos equipos y dar por terminado al proyecto cooperativo.
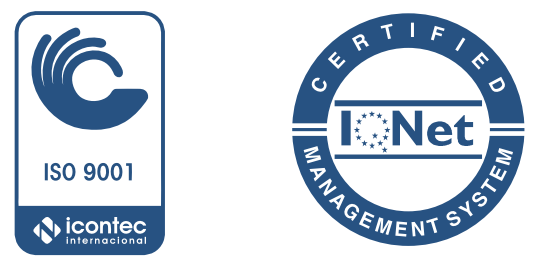


\section{CONCLUSIONES}

La comunicación sincera, periódica y positiva es un factor clave para la realización de proyectos de cooperación internacional, y en este caso permitió la definición del proceso estandarizado para el ensamble del carro a escala Jeep Wrangler 2018.

Es importante generar un pensamiento crítico a las diversas interpretaciones de la realidad, por esto es importante que los miembros de cada equipo escuchen, analicen, interpreten y tengan mente abierta a los mensajes y observaciones recibidos.

Tanto el grupo de Costa Rica como el grupo de Colombia, tuvo una aceptación con respecto a la facilidad de adaptación durante el proceso.
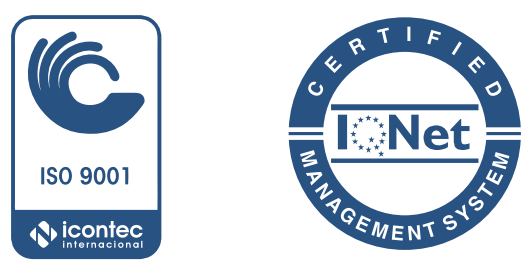


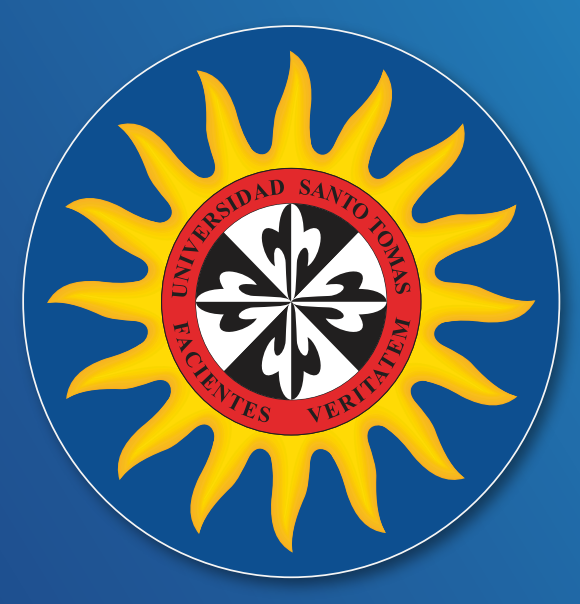

\section{UNIVERSIDAD SANTO TOMÁS} PRIMER CLAUSTRO UNIVERSITARIO DE COLOMBIA FACULTAD DE INGENIERIA INDUSTRIAL
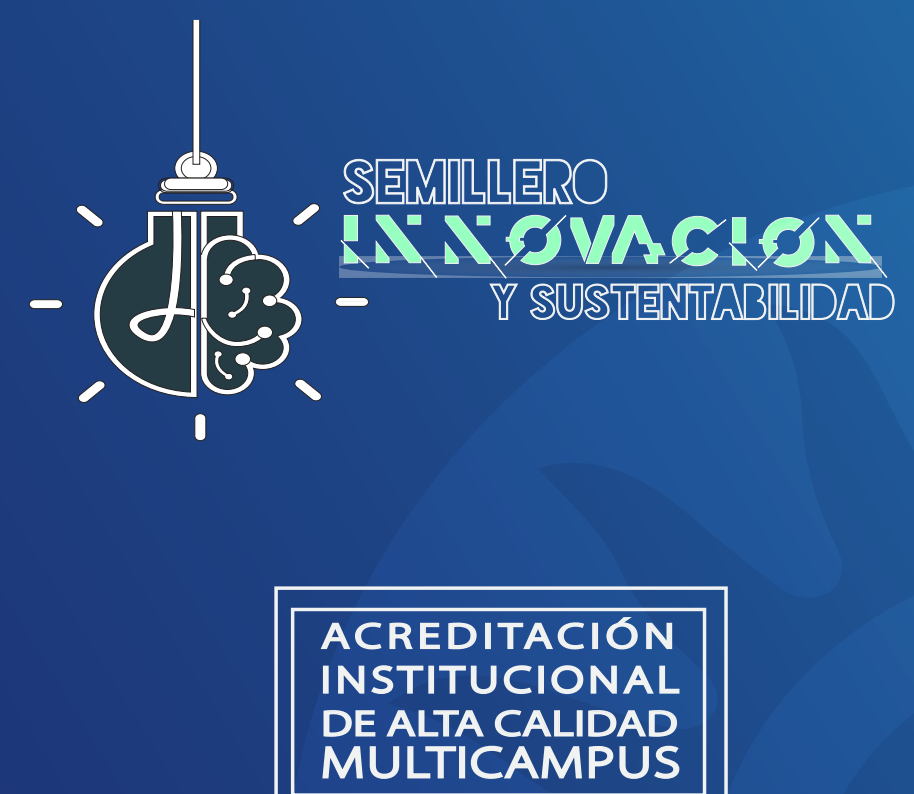

Res MEN No 01458 del 29 de enero de 2016
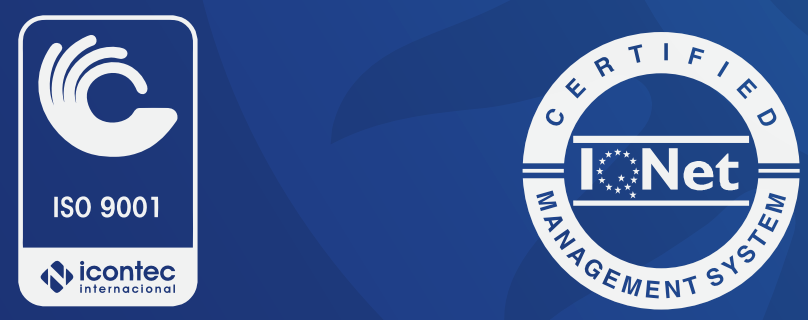